\title{
New round of research grant applications launched
}

Dental academics and clinicians are invited to apply for research grants from Foundation Nakao. This is the second round of funding made available by this foundation since its launch in 2018 , supporting clinical trials and research into important subjects such as minimum intervention dentistry and oral health of the elderly.

Successful applications will receive fully funded projects of CHF 50,000 [about $£ 42,000$ ] per project in addition to wide exposure among dental professionals, the dental industry as well as the general public, of each study's outcomes and achievements.

The Foundation's first round of grant applications took place in September 2019 and six studies were awarded the honour of being accepted out of a huge number of submissions. Applicants represented the categories: government organisation, non-government organisation, university, research institution or other.

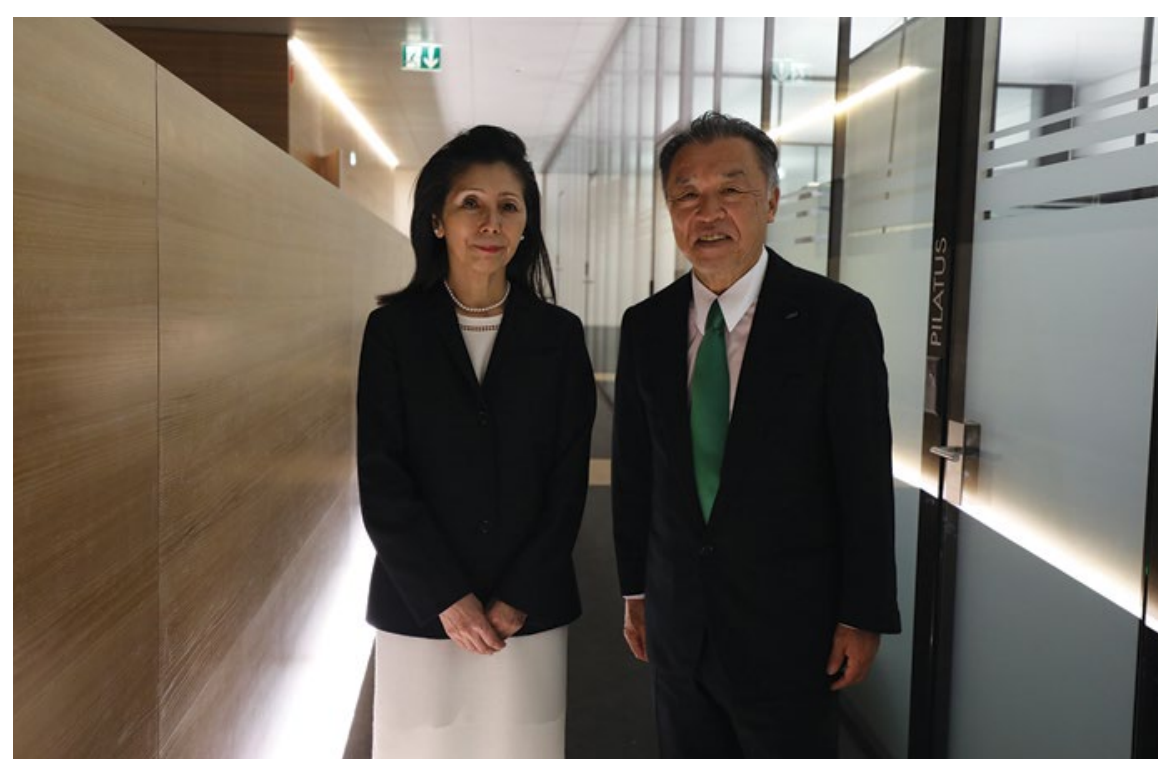

Mrs Makiko Nakao, President of the Foundation Board and Mr Makoto Nakao
Foundation Nakao supports academic research and clinical studies contributing to its founding goal, which is the improvement of oral health and subsequent raised quality of life of all people around the world. Key oral health research areas address minimum intervention dentistry, oral health in ageing populations and the 8020 movement, tooth function, the prevention of oral frailty and dental IQ.

Foundation Nakao for Worldwide Oral Health was launched on 21 September 2018 in Luzern, Switzerland. It was made possible by Mr Makoto Nakao, former Chairman of the GC Corporation, who after 42 years at the helm of the company donated his privately-owned company shares to support this initiative.

The Foundation's management board boasts a team of distinguished dental professionals from four continents: Europe, America, Australia and Asia. They are Professor Reinhart Hickel, Professor Clark Stanford, Professor Macro Ferrari, Professor Eric Reynolds, Professor Keiichi Sasaki and Dr Kiyotaka Nakao.

Grant applications are open until 11 December 2020 via the application form on the Nakao Foundation website www. foundation-nakao.com/applications.

\section{Stylish and eco-conscious PPE made on a farm}

Scrubs in Style is a brand new company created during lockdown by two sisters with very different careers: Diane is a dental hygienist and Kate is a bespoke dressmaker.

Inspiration behind their work came from discovering that PPE initially was difficult to get hold of during the pandemic, uncomfortable to wear, looked awful, created mountains of plastic waste and was expensive to sustain.

Scrubs in Style make all the PPE themselves from their workshop on a farm in Oxfordshire.

Diane and Kate have combined their skills to produce the best quality washable/ sterilisable gowns and aprons. Their emphasis is on helping to reduce plastic waste and injecting style and comfort into the PPE with ultimate protection.

Scrubs in Style's gowns are made from UK and specialist German sourced material. The fabric is colour fast, shrink resistant and does not bleed; it's also cool and comfortable to wear. With 20 colours to choose from there is something for everyone. PPE aprons are made from the same material in white. PPE can be wiped down with a disinfectant or washed at temperatures up to 90 degrees and sterilised.

For more information email scrubsinstyle.info@gmail.com. If you would like to see samples of the range so far, Diane and Kate would be happy to arrange a visit/Zoom.

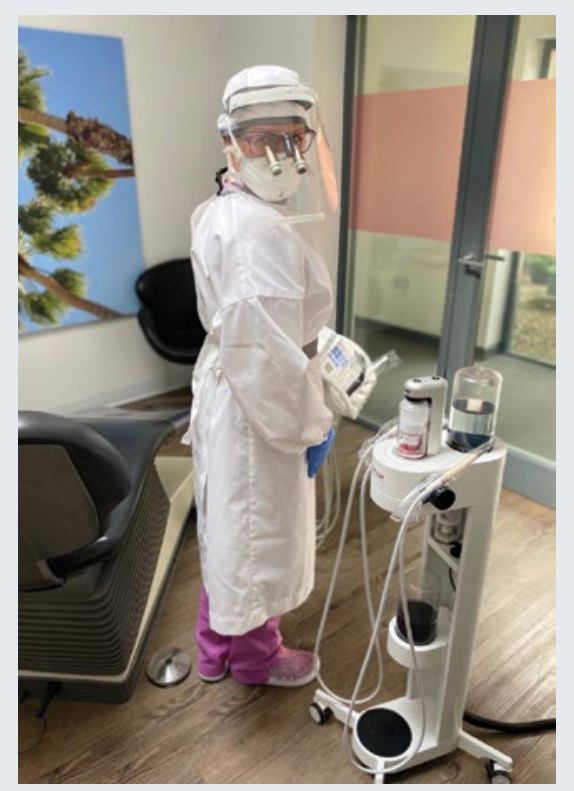

